\title{
Open versus percutaneous instrumentation in thoracolumbar fractures: magnetic resonance imaging comparison of paravertebral muscles after implant removal
}

\author{
Yves Ntilikina, MD, David Bahlau, MD, Julien Garnon, MD, Sébastien Schuller, MD, \\ Axel Walter, MD, Mickaël Schaeffer, MSc, Jean-Paul Steib, PhD, and Yann Philippe Charles, PhD
}

Service de Chirurgie du Rachis, Hôpitaux Universitaires de Strasbourg, Fédération de Médecine Translationnelle (FMTS), Université de Strasbourg, France

OBJECTIVE Percutaneous instrumentation in thoracolumbar fractures is intended to decrease paravertebral muscle damage by avoiding dissection. The aim of this study was to compare muscles at instrumented levels in patients who were treated by open or percutaneous surgery.

METHODS Twenty-seven patients underwent open instrumentation, and 65 were treated percutaneously. A standardized MRI protocol using axial T1-weighted sequences was performed at a minimum 1-year follow-up after implant removal. Two independent observers measured cross-sectional areas (CSAs, in $\mathrm{cm}^{2}$ ) and region of interest (ROI) signal intensity (in pixels) of paravertebral muscles by using OsiriX at the fracture level, and at cranial and caudal instrumented pedicle levels. An interobserver comparison was made using the Bland-Altman method. Reference ROI muscle was assessed in the psoas and ROI fat subcutaneously. The ratio ROI-CSA/ROI-fat was compared for patients treated with open versus percutaneous procedures by using a linear mixed model. A linear regression analyzed additional factors: age, sex, body mass index (BMI), Pfirrmann grade of adjacent discs, and duration of instrumentation in situ.

RESULTS The interobserver agreement was good for all CSAs. The average CSA for the entire spine was $15.7 \mathrm{~cm}^{2}$ in the open surgery group and $18.5 \mathrm{~cm}^{2}$ in the percutaneous group $(p=0.0234)$. The average ROI-fat and ROI-muscle signal intensities were comparable: 497.1 versus 483.9 pixels for ROI-fat and 120.4 versus 111.7 pixels for ROI-muscle in open versus percutaneous groups. The ROI-CSA varied between 154 and 226 for open, and between 154 and 195 for percutaneous procedures, depending on instrumented levels. A significant difference of the ROI-CSA/ROI-fat ratio $(0.4$ vs 0.3$)$ was present at fracture levels T12-L1 $(p=0.0329)$ and at adjacent cranial $(p=0.0139)$ and caudal $(p=0.0100)$ instrumented levels. Differences were not significant at thoracic levels. When adjusting based on age, BMI, and Pfirrmann grade, a significant difference between open and percutaneous procedures regarding the ROI-CSA/ROI-fat ratio was present in the lumbar spine ( $p$ 0.01). Sex and duration of instrumentation had no significant influence.

CONCLUSIONS Percutaneous instrumentation decreased muscle atrophy compared with open surgery. The MRI signal differences for T-12 and L-1 fractures indicated less fat infiltration within CSAs in patients who received percutaneous treatment. Differences were not evidenced at thoracic levels, where CSAs were smaller. Fat infiltration was not significantly different at lumbar levels with either procedure in elderly patients with associated discopathy and higher BMI. In younger patients, there was less fat infiltration of lumbar paravertebral muscles with percutaneous procedures.

https://thejns.org/doi/abs/10.3171/2017.1.SPINE16886

KEY WORDS thoracolumbar fracture; minimally invasive surgery; percutaneous instrumentation; paravertebral muscle atrophy; fat infiltration; MRI; trauma

ABBREVIATIONS BMI = body mass index; $C S A=$ cross-sectional area; $M I S=$ minimally invasive surgery; $R O I=$ region of interest.

SUBMITTED July 29, 2016. ACCEPTED January 5, 2017.

INCLUDE WHEN CITING Published online June 9, 2017; DOI: 10.3171/2017.1.SPINE16886. 
$\mathrm{M}$ INIMALLY invasive surgery (MIS) has become a valuable option in the treatment of thoracolumbar trauma. ${ }^{25}$ Percutaneous instrumentation lowers intraoperative blood loss, risk for infection, and postoperative pain, and shortens hospital stay compared with conventional surgery. ${ }^{5}$ These advantages of posterior instrumentation through MIS access are clinically and economically relevant in the short term. Posttraumatic kyphosis correction seems as effective with modern percutaneous techniques as reduction obtained by conventional instrumentation, thus providing adequate sagittal balance and satisfying long-term results. ${ }^{3,9,24}$

Percutaneous instrumentation represents an efficient reduction tool, and it is eventually combined with cement augmentation of the vertebral body ${ }^{1}$ or an additional anterior graft, depending on the fracture type. In cases of selective anterior fusion, posterior instrumentation may be used as a temporary internal fixator, which is removed once the fracture is healed or fusion is obtained. ${ }^{6,27}$ Minimally invasive surgery seems particularly interesting with regard to the paravertebral muscles because dissection is avoided, which might improve postoperative rehabilitation after implant removal. It has been reported that short-segment MIS instrumentation would provide less muscle atrophy and fat infiltration compared with open surgery. ${ }^{13,19}$ This seems valid for certain parts of the musculature only when using muscle-sparing techniques in the lumbar spine. ${ }^{23}$ Muscle atrophy and fat infiltration have not been analyzed in the entire thoracolumbar spine for traumatic indications, and to our knowledge the theoretical advantage of MIS over conventional surgery has not been verified on MRI so far.

The purpose of this study was to compare paravertebral muscles in patients with thoracolumbar fracture treated by open or percutaneous surgery. A standardized MRI protocol was used at a minimum 1-year follow-up after implant removal to quantify differences in muscle atrophy and fat infiltration. The influence of patient-related factors was additionally taken into account.

\section{Methods}

A retrospective study was conducted in patients with nonneurological thoracolumbar fractures at our institution. Between 2008 and 2010, 59 consecutive patients were treated by open surgery, which was still our standard at that time. Between 2010 and 2012, 129 patients underwent percutaneous procedures, which became our standard after 2010. Open and percutaneous instrumentations were performed for similar fracture types. Only patients who underwent implant removal after fracture consolidation, and who agreed to have MRI after informed consent, were enrolled in the study protocol. Patients with anterior cages producing metallic artifacts were excluded. There were 27 patients in the open instrumentation group and 65 in the percutaneous instrumentation group. Demographic data including sex, age at surgery, body mass index (BMI), duration of instrumentation in situ, fracture levels, and fracture types according to the $\mathrm{AO}$ classification ${ }^{16}$ are summarized in Table 1.

A standardized MRI protocol was applied to each pa-
TABLE 1. Demographic data of 92 patients with thoracolumbar fractures in open and percutaneous surgery groups

\begin{tabular}{lcc}
\hline \multicolumn{1}{c}{ Characteristic } & Open, $\mathrm{n}=27$ & Percutaneous, $\mathrm{n}=65$ \\
\hline Sex & $20 \mathrm{M}, 7 \mathrm{~F}$ & $36 \mathrm{M}, 29 \mathrm{~F}$ \\
\hline Age & $43.2 \mathrm{yrs}(20-71)$ & $48.5 \mathrm{yrs}(16-75)$ \\
\hline BMI & $23.5(17-32)$ & $25.9(16-39)$ \\
\hline Instrumentation duration & $17.6 \mathrm{mos}(8-46)$ & 14.4 mos $(6-35)$ \\
\hline Fracture levels & T8-L3 & T7-L5 \\
\hline $\begin{array}{l}\text { Fracture types-AO } \\
\text { system }\end{array}$ & 9 Type A2.3 & 21 Type A2.3 \\
& 12 Type A3.2 or A3.3 & 32 Type A3.2 or A3.3 \\
& 6 Type B2.1 or B2.2 & 12 Type B2.1 or B2.2 \\
\hline
\end{tabular}

Values in parentheses represent ranges.

tient (Siemens Aera 1.5 T). Axial T1-weighted images (slice thickness $1 \mathrm{~mm}$, TR $521.0 \mathrm{msec}$, TE $8.5 \mathrm{msec}$ ) were analyzed at the pedicle level of the fractured vertebra and at pedicles that had been instrumented with screws, including 1 or 2 cranial and caudal adjacent levels, depending on length of instrumentation (Fig. 1). Images were then processed with OsiriX 3.9.2 software (Pixmeo). Crosssectional areas (CSAs, in $\mathrm{cm}^{2}$ ) of paravertebral muscles were assessed at each level on the right and left sides. The CSAs were manually contoured with the software (Fig. 2). Two independent raters performed measurements at each level, to assess the concordance between both raters by using the Bland-Altman method. A good interrater agreement was demonstrated for all CSAs, thus validating the accuracy of the method.

The CSA values were further used to compare the amount of muscle atrophy in percutaneous and open procedure groups. The average signal intensities were then calculated in pixels within regions of interest (ROIs) to determine the amount of fat infiltration within the CSA. The reference ROI of muscle was assessed in the psoas and the ROI of fat was assessed subcutaneously. The ROI signal intensity for paravertebral musculature was calculated as an average over the entire CSA. Scar tissue was characterized by a signal intensity that was similar to fat and easily distinguished from low muscle signal intensity. Therefore, scar tissue was assimilated to fat infiltration. For further evaluation, the ratio ROI-CSA/ROI-fat was considered. This ratio increases as the amount of fat infiltration within CSA increases. Additionally, degenerative changes of intervertebral discs adjacent to the fracture level were rated on sagittal T2-weighted sequences according to Pfirrmann et al. ${ }^{21}$ and grouped in Grades I-III (minor degeneration) and Grades IV-V (severe degeneration).

Statistical analysis was performed with $\mathrm{R}$ software version 3.1.0 (R Foundation). A Shapiro-Wilk test was used to check normal distribution of ROI-CSA in both groups. Because measurements were done by 2 independent raters, 54 measurements were considered in the open group and 130 in the percutaneous group. Ratios ROI-CSA/ROI-fat were compared at fracture, and at caudal and cranial adjacent levels between open and percutaneous groups, using mixed models. Thus intraclass variances across a random rater effect and a random subject effect were taken into account. 

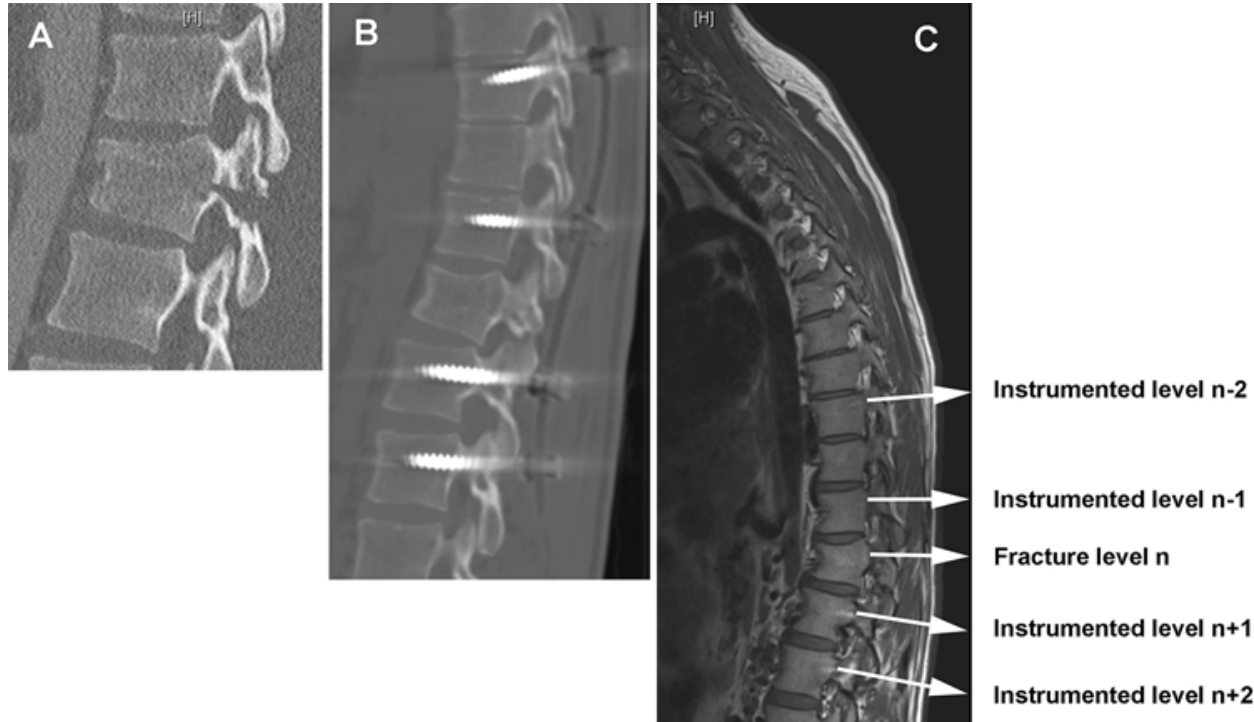

FIG. 1. A: Sagittal CT scan of an L-1 fracture. B: The same fracture after percutaneous instrumentation. C: A T1-weighted MR image obtained after implant removal: the fracture level and instrumented levels are indicated by arrows.

A multivariate model was then fitted, including adjustments on potential bias factors that might influence the ratio ROI-CSA/ROI-fat: age, sex, BMI, disc degeneration, and duration of instrumentation in situ. Because of anatomical differences in paravertebral muscle CSAs, thoracic and lumbar areas were evaluated separately. The ROI-muscle was further analyzed with a linear model to measure the correlation with disc degeneration. The significance level was set at $p=0.05$.

\section{Results}

\section{Cross-Sectional Areas}

The average CSA was $11.5 \pm 6.2 \mathrm{~cm}^{2}$ (range 1.6-33.3 $\mathrm{cm}^{2}$ ) in the thoracic spine and $19.2 \pm 7.4 \mathrm{~cm}^{2}$ (range 3.1$\left.43.9 \mathrm{~cm}^{2}\right)$ in the lumbar spine. This difference between thoracic and lumbar spine was significant $(\mathrm{p}<0.0001)$. The average CSA for the entire spine was $15.7 \pm 7.7 \mathrm{~cm}^{2}$ (range 1.6-37.9 $\mathrm{cm}^{2}$ ) in the open group and $18.5 \pm 7.7 \mathrm{~cm}^{2}$ (range $3.1-43.9 \mathrm{~cm}^{2}$ ) in the percutaneous group. This difference was also significant when adjusting on similar spinal levels $(\mathrm{p}=0.0234)$.

\section{Signal Intensities on MRI}

Table 2 shows global results of the cohort at thoracic and lumbar levels. The average ROI-fat and ROI-muscle were comparable in open versus percutaneous groups. The ratios ROI-CSA/ROI-fat were 0.4 in open and 0.3 in percutaneous groups.

When comparing open and percutaneous groups, a significant difference of the ROI-CSA/ROI-fat ratio was
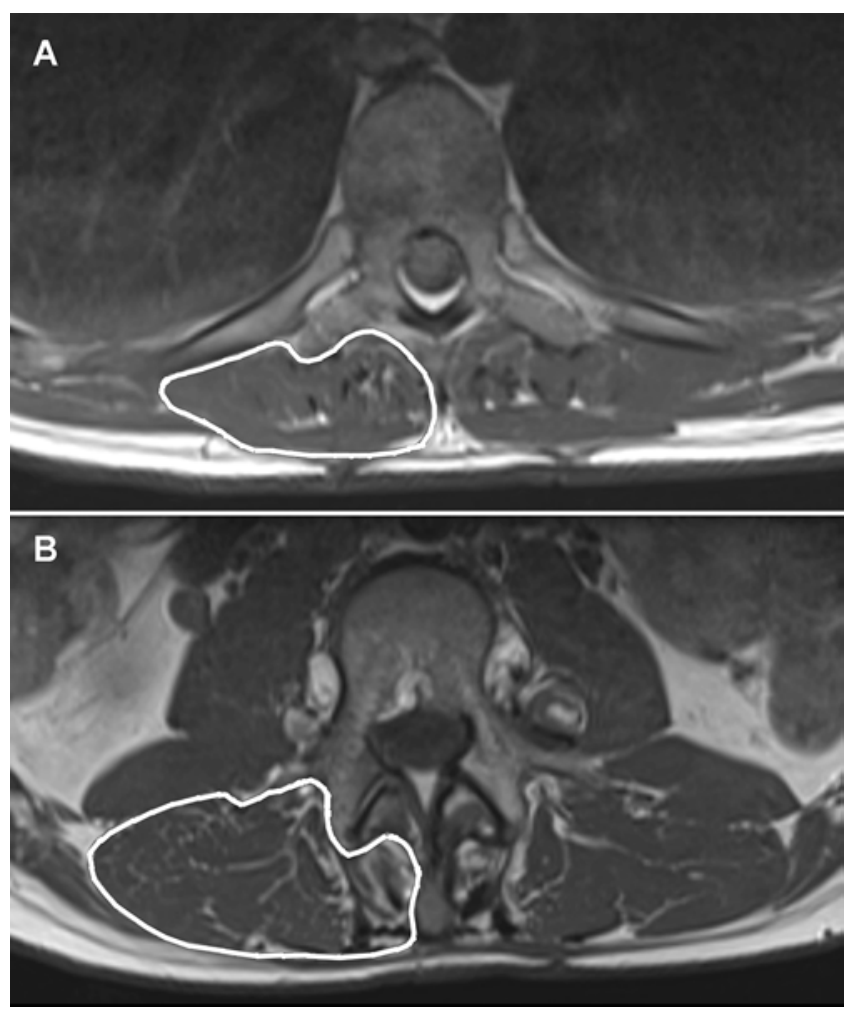

FIG. 2. Axial cuts of the lumbar spine at the pedicle level on a T1-weighted MR image: paravertebral muscles are contoured with a mouse-guided tool. 
TABLE 2. Signal intensities in the ROI and ROI-CSA/ROI-fat ratio in patients with thoracolumbar fractures

\begin{tabular}{|c|c|c|}
\hline \multirow[b]{2}{*}{ Variable } & \multicolumn{2}{|c|}{ MRI Signal Intensity Postop } \\
\hline & Open & Percutaneous \\
\hline $\begin{array}{l}\text { ROl-fat, subcuta- } \\
\text { neous }\end{array}$ & $497.1 \pm 61.2(273-828)$ & $483.9 \pm 69.5(169-650)$ \\
\hline ROI-muscle, psoas & $120.4 \pm 97.7(52-605)$ & $111.7 \pm 38.7(47-189)$ \\
\hline $\begin{array}{l}\text { ROI-CSA, paraver- } \\
\text { tebral }\end{array}$ & $185.5 \pm 69.5(154-226)$ & $173.9 \pm 38.0(154-195)$ \\
\hline $\begin{array}{l}\text { Ratio ROI-CSA/ } \\
\text { ROI-fat }\end{array}$ & $0.4 \pm 0.1(0.1-0.8)$ & $0.3 \pm 0.1(0.1-0.6)$ \\
\hline
\end{tabular}

Values of ROls are expressed in pixels, as the mean \pm SD (range).

present at fracture levels T-12 and L-1 $(\mathrm{p}=0.0329)$, and at cranial $(\mathrm{p}=0.0139)$ and caudal $(\mathrm{p}=0.0100)$ adjacent instrumented levels. Differences were not significant at other thoracic or lumbar fracture levels when considering global results without adjustment of associated factors.

\section{Influence of Associated Factors}

Additional factors that might affect the amount of fatty infiltration of the paravertebral muscles were investigated (Figs. 3 and 4). Age had a significant influence on the ratio ROI-CSA/ROI-fat for the thoracolumbar junction and lumbar levels ( $\mathrm{p}<0.0001)$. The same applied for BMI ( $\mathrm{p}$ $<0.0001$ ). Duration of instrumentation in situ and sex had no significant influence on the ratio.

Additionally, the correlation between signal intensity of ROI muscle (psoas) and disc degeneration (Pfirrmann Grades I-III versus Grades IV-V) was analyzed. There was a significant correlation between the grade of fatty infiltration within the psoas and disc degeneration at caudal instrumented levels $n+1(p=0.0478)$ and $n+2(p=$ 0.0060).

When using a multivariate model implementing age, BMI, and Pfirrmann grade, there were significant differences of ROI-CSA/ROI-fat ratios between open and percutaneous groups in the lumbar spine only (Table 3 ). This indicated that younger patients with lower BMI and minor disc degeneration would have less fatty infiltration of their lumbar paravertebral muscles when undergoing percutaneous operation. There would be minor differences in muscle dystrophy between open and percutaneous procedures in elderly patients with higher BMI and associated disc degeneration. No differences were found at thoracic levels regardless of associated factors.

\section{Discussion}

Open instrumentation used to be considered the gold standard procedure for unstable thoracolumbar fractures, but it is known that paravertebral muscle function might be altered after a posterior approach. ${ }^{26}$ Kawaguchi et al. ${ }^{11,12}$ analyzed the microscopic and biochemical effects of posterior open spine surgery on paravertebral muscles. They demonstrated that pressure caused by retractors and duration of surgery were correlated with the amount of muscle injury. Mayer et al. ${ }^{17}$ studied psoas and paravertebral muscle density by using a CT protocol 3 months after open surgery. They showed that there was a significant decrease in muscle density of the psoas and erector spinae, interpreted as atrophy, and a trend toward decreased CSAs. They further showed that isokinetic trunk strength and greater muscle density on CT were correlated. Magnetic resonance imaging represents a reliable tool to evaluate the amount of fat infiltration of paravertebral muscles. ${ }^{18}$
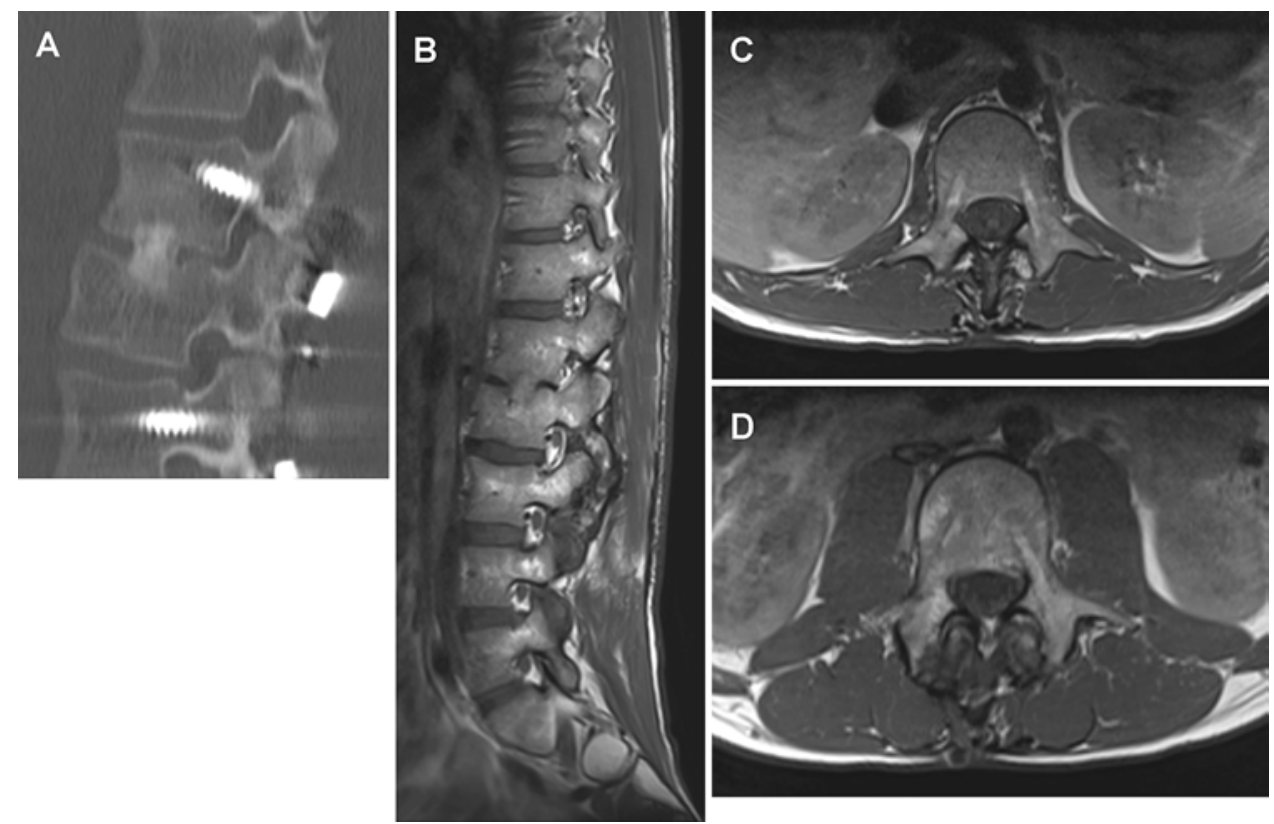

FIG. 3. A 29-year-old man with an AO Type A2.3 fracture of L-2. A: Sagittal CT scan of L1-3 arthrodesis after open surgery. B: Sagittal T1-weighted MR image of the same fracture after implant removal. C and D: Axial T1-weighted MR images of the same fracture after implant removal: paravertebral muscles seem to have a small amount of fatty infiltration. 

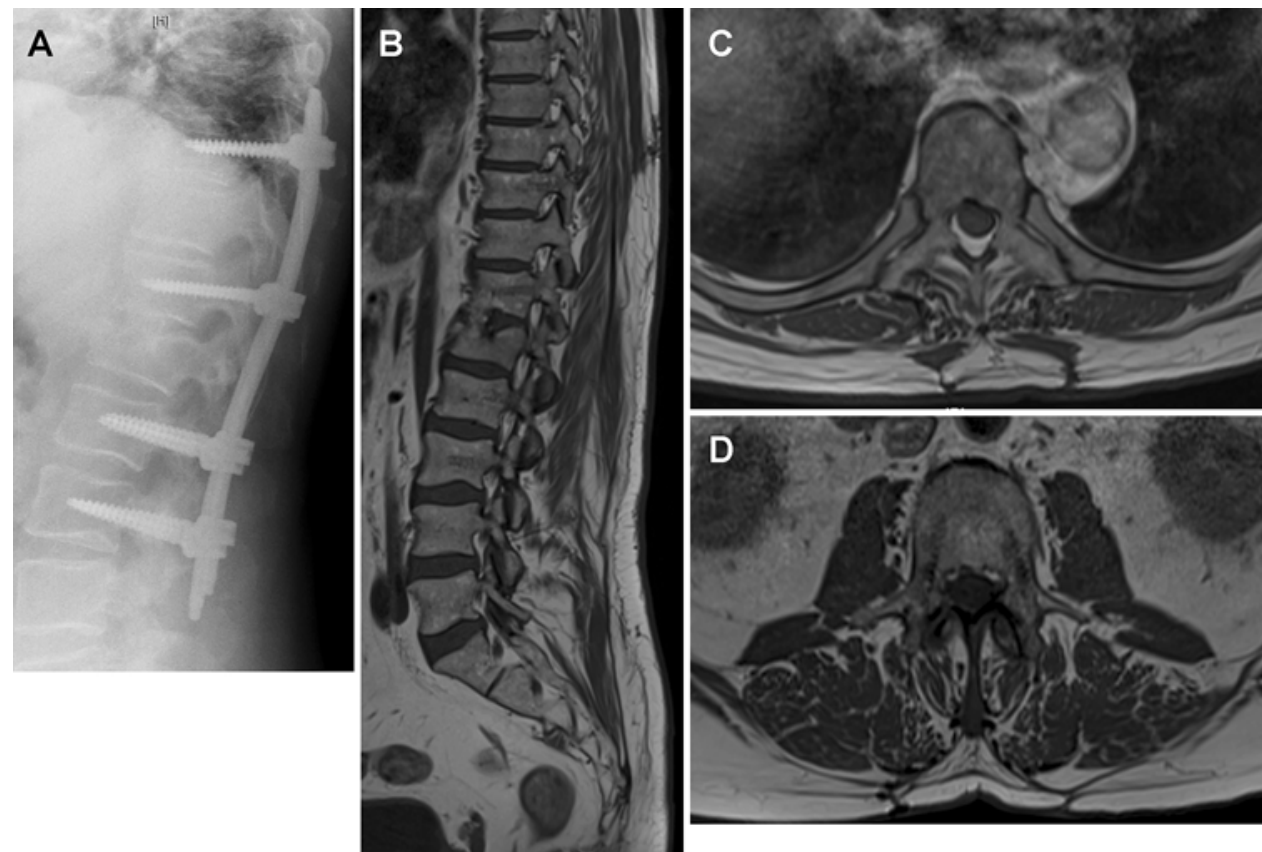

FIG. 4. A 68-year-old man with an AO Type A3.2 fracture of L-1. A: Sagittal CT scan of T10-L4 percutaneous instrumentation. B: Sagittal T1-weighted MR image of the same fracture after implant removal. C and D: Axial T1-weighted MR images of the same fracture after implant removal: paravertebral muscles have a large amount of fatty infiltration.

Ghiasi et al. ${ }^{7}$ used an MRI protocol 6 months after open posterior lumbar instrumentation. There was a significant decrease of CSA of erector spinae and multifidus muscles. An increase of fat infiltration and noncontractile connective muscle fibers within the CSA was found to be significantly higher at lower lumbar levels (L4-5 and L5-S1). This is in line with the findings of Gille et al., ${ }^{8}$ who analyzed the paraspinal musculature on MRI after L4-5 open posterior fusion 6 months postoperatively. These authors demonstrated that fatty involution of the erector spinae was negligible proximally, whereas it was more marked distally in relation to the level of arthrodesis.

Over the last decade, there is a trend toward more indications for MIS in thoracolumbar instrumentation, because these procedures reduce blood loss, postoperative pain, and length of hospital stay. ${ }^{1,3,5,9,24,25} \mathrm{Kim}$ et al. $.^{14} \mathrm{com}-$ pared trunk muscle strength between patients treated with open posterior (8 patients) versus percutaneous lumbar instrumentation (11 patients) by using functional tests and MRI. Patients who underwent percutaneous instrumentation displayed higher isometric trunk extension strength compared with patients who underwent open operations. Extension strength seemed to correlate with preservation of multifidus muscles as measured on CSA. Although the subgroups compared in this study were small, these observations might sustain the theory that percutaneous surgery would better preserve the paravertebral musculature.

Hyun et al. ${ }^{10}$ compared paravertebral muscle atrophy on CT after paramedian interfascial versus traditional midline approaches in 26 patients. The CSA of the multifidus muscle was higher when using the interfascial paramedian approach. Fat infiltration was not evaluated because of metallic artifacts of instrumentation. Min et al. ${ }^{19} \mathrm{com}-$ pared paravertebral muscles after MIS versus open transforaminal lumbar interbody fusion on pre- and postoperative MRI. In single-level MIS procedures, fat infiltration

TABLE 3. Comparison of open versus percutaneous instrumentation adjusted based on age, BMI, and disc degeneration in a multivariate model in patients with thoracolumbar fractures

\begin{tabular}{|c|c|c|c|c|c|c|}
\hline \multirow{2}{*}{$\begin{array}{c}\text { Instrumented } \\
\text { Level }\end{array}$} & \multicolumn{2}{|c|}{ Thoracic Muscles } & \multirow[b]{2}{*}{$p$ Value } & \multicolumn{2}{|c|}{ Lumbar Muscles } & \multirow[b]{2}{*}{$p$ Value } \\
\hline & Open & Percutaneous & & Open & Percutaneous & \\
\hline \multicolumn{7}{|l|}{ Cranial } \\
\hline$n-2$ & $0.34 \pm 0.15$ & $0.33 \pm 0.07$ & 0.8342 & $0.46 \pm 0.19$ & $0.32 \pm 0.08$ & 0.0035 \\
\hline$n-1$ & $0.37 \pm 0.15$ & $0.32 \pm 0.05$ & 0.9880 & $0.45 \pm 0.17$ & $0.35 \pm 0.08$ & 0.0165 \\
\hline \multicolumn{7}{|l|}{ Caudal } \\
\hline$n+1$ & $0.36 \pm 0.13$ & $0.33 \pm 0.06$ & 0.9948 & $0.47 \pm 0.17$ & $0.33 \pm 0.09$ & 0.0065 \\
\hline$n+2$ & $0.36 \pm 0.10$ & $0.32 \pm 0.07$ & 0.8813 & $0.44 \pm 0.21$ & $0.40 \pm 0.09$ & 0.0046 \\
\hline
\end{tabular}

Values are expressed as the mean $\pm \mathrm{SD}$ of the ROI-CSA/ROI-fat ratio. Boldface type indicates statistical significance. 
increased by $1.37 \%$, versus $4.30 \%$ with open surgery. Relative CSA decreased by 0.07 versus 0.10 , respectively. In multisegment instrumentation, fat infiltration increased by $2.79 \%$ for MIS versus $7.90 \%$ for open procedures. Relative CSA decreased by 0.10 versus 0.16 , respectively. After a period of more than 1 year, there was no significant difference in paravertebral muscle atrophy and fat infiltration between the 2 techniques. In our cohort of patients with thoracolumbar trauma, the CSA was globally smaller after open compared with percutaneous instrumentation. When considering fat infiltration, the global difference between open and percutaneous procedures was relatively low. However, additional factors other than surgical access might influence the amount of muscle atrophy and fat infiltration, which has been studied in detail.

Age represents one factor that influences muscle dystrophy after spinal surgery. Takayama et al. ${ }^{28}$ demonstrated that the CSA of paravertebral muscles on MRI was significantly lower in subjects older than 60 years and in subjects younger than 20 years, thus implying that paravertebral muscle atrophy was related to age. Furthermore, the fat infiltration rate increased with age, most markedly at the lower lumbar levels. Chen et al. ${ }^{4}$ also demonstrated that multifidus fat infiltration was prominently found in elderly patients with lumbar spinal stenosis. Parkkola et al. ${ }^{20}$ compared lumbar intervertebral discs, fat infiltration, and CSA of psoas and paravertebral muscles in middle-aged healthy volunteers and in patients presenting with low-back pain. Disc degeneration was more frequently seen in the patients than in the healthy volunteers. The CSAs of psoas and paravertebral muscles in patients with low-back pain were smaller than those of healthy volunteers. Patients with low-back pain furthermore presented more fat infiltration of muscles than did controls. Ploumis et al. ${ }^{22}$ reported a similar relationship between discopathy and muscle atrophy. Lee et al. ${ }^{15}$ quantified the percentage of fat infiltration within the CSA of lumbar paravertebral muscles: $41.3 \%$ in patients with degenerative flat back versus $15.9 \%$ in healthy controls. In our study, we demonstrated that age, grade of disc degeneration, and BMI were related to the amount of paravertebral muscle fat infiltration, which is in line with these previous findings. To assess the effect of percutaneous versus open instrumentation on paravertebral muscles, it appeared essential to adjust the statistical analysis based on these influencing parameters. A statistically significant advantage of percutaneous instrumentation on fat infiltration of paravertebral muscles was only evidenced in younger patients at the thoracolumbar junction and lumbar levels.

Our study presents some limitations because it is retrospective, and preoperative MRI data were not available, which would enable pre- and postoperative comparisons. Furthermore, our results are purely morphological, analyzing fat infiltration on MRI. Functional results such as muscle strength testing were not assessed. However, Cawley et al. ${ }^{2}$ studied paravertebral muscle CSA by using ultrasonography and muscle innervation by using electromyography in patients with lumbar fractures. A better innervation and greater CSA of the multifidus muscle was observed in 6 patients treated by MIS compared with 6 patients who received open instrumentation.

\section{Conclusions}

Percutaneous instrumentation decreased muscle atrophy compared with open surgery. The MRI signal differences were evidenced for T-12 and L-1 fractures, indicating less fat infiltration within the CSA in patients treated percutaneously. Differences between MIS and open surgery were not evidenced at thoracic levels, where CSAs were smaller. In the lumbar spine, fat infiltration was influenced by age, discopathy, and BMI. Younger patients without degenerative changes and with a lower BMI presented significantly less paravertebral fat infiltration after MIS.

\section{References}

1. Blondel B, Fuentes S, Pech-Gourg G, Adetchessi T, Tropiano P, Dufour H: Percutaneous management of thoracolumbar burst fractures: evolution of techniques and strategy. Orthop Traumatol Surg Res 97:527-532, 2011

2. Cawley DT, Alexander M, Morris S: Multifidus innervation and muscle assessment post-spinal surgery. Eur Spine J 23:320-327, 2014

3. Charles YP, Zairi F, Vincent C, Fuentes S, Bronsard N, Court $\mathrm{C}$, et al: Minimally invasive posterior surgery for thoracolumbar fractures. New trends to decrease muscle damage. Eur J Orthop Surg Traumatol 12:1-7, 2011

4. Chen YY, Pao JL, Liaw CK, Hsu WL, Yang RS: Image changes of paraspinal muscles and clinical correlations in patients with unilateral lumbar spinal stenosis. Eur Spine J 23:999-1006, 2014

5. Court C, Vincent C: Percutaneous fixation of thoracolumbar fractures: current concepts. Orthop Traumatol Surg Res 98:900-909, 2012

6. Eck JC: Minimally invasive corpectomy and posterior stabilization for lumbar burst fracture. Spine J 11:904-908, 2011

7. Ghiasi MS, Arjmand N, Shirazi-Adl A, Farahmand F, Hashemi $\mathrm{H}$, Bagheri S, et al: Cross-sectional area of human trunk paraspinal muscles before and after posterior lumbar surgery using magnetic resonance imaging. Eur Spine J 25:774-782, 2016

8. Gille O, Jolivet E, Dousset V, Degrise C, Obeid I, Vital JM, et al: Erector spinae muscle changes on magnetic resonance imaging following lumbar surgery through a posterior approach. Spine (Phila Pa 1976) 32:1236-1241, 2007

9. Grass R, Biewener A, Dickopf A, Rammelt S, Heineck J, Zwipp H: Perkutane dorsale versus offene Instrumentation bei Frakturen des thorakolumbalen Ubergangs. Eine vergleichende prospektive Untersuchung. Unfallchirurg 109:297305, 2006

10. Hyun SJ, Kim YB, Kim YS, Park SW, Nam TK, Hong HJ, et al: Postoperative changes in paraspinal muscle volume: comparison between paramedian interfascial and midline approaches for lumbar fusion. J Korean Med Sci 22:646-651, 2007

11. Kawaguchi Y, Matsui H, Tsuji H: Back muscle injury after posterior lumbar spine surgery. A histologic and enzymatic analysis. Spine (Phila Pa 1976) 21:941-944, 1996

12. Kawaguchi Y, Matsui H, Tsuji H: Back muscle injury after posterior lumbar spine surgery. Part 2: Histologic and histochemical analyses in humans. Spine (Phila Pa 1976) 19:2598-2602, 1994

13. Kim CW: Scientific basis of minimally invasive spine surgery: prevention of multifidus muscle injury during posterior lumbar surgery. Spine (Phila Pa 1976) 35 (26 Suppl):S281S286, 2010

14. Kim DY, Lee SH, Chung SK, Lee HY: Comparison of multifidus muscle atrophy and trunk extension muscle strength: percutaneous versus open pedicle screw fixation. Spine (Phila Pa 1976) 30:123-129, 2005 
15. Lee JC, Cha JG, Kim Y, Kim YI, Shin BJ: Quantitative analysis of back muscle degeneration in the patients with the degenerative lumbar flat back using a digital image analysis: comparison with the normal controls. Spine (Phila Pa 1976) 33:318-325, 2008

16. Magerl F, Aebi M, Gertzbein SD, Harms J, Nazarian S: A comprehensive classification of thoracic and lumbar injuries. Eur Spine J 3:184-201, 1994

17. Mayer TG, Vanharanta H, Gatchel RJ, Mooney V, Barnes D, Judge L, et al: Comparison of CT scan muscle measurements and isokinetic trunk strength in postoperative patients. Spine (Phila Pa 1976) 14:33-36, 1989

18. Mhuiris AN, Volken T, Elliott JM, Hoggarth M, Samartzis D, Crawford RJ: Reliability of quantifying the spatial distribution of fatty infiltration in lumbar paravertebral muscles using a new segmentation method for T1-weighted MRI. BMC Musculoskelet Disord 17:234, 2016

19. Min SH, Kim MH, Seo JB, Lee JY, Lee DH: The quantitative analysis of back muscle degeneration after posterior lumbar fusion: comparison of minimally invasive and conventional open surgery. Asian Spine J 3:89-95, 2009

20. Parkkola R, Rytökoski U, Kormano M: Magnetic resonance imaging of the discs and trunk muscles in patients with chronic low back pain and healthy control subjects. Spine (Phila Pa 1976) 18:830-836, 1993

21. Pfirrmann CW, Metzdorf A, Zanetti M, Hodler J, Boos N: Magnetic resonance classification of lumbar intervertebral disc degeneration. Spine (Phila Pa 1976) 26:1873-1878, 2001

22. Ploumis A, Michailidis N, Christodoulou P, Kalaitzoglou I, Gouvas G, Beris A: Ipsilateral atrophy of paraspinal and psoas muscle in unilateral back pain patients with monosegmental degenerative disc disease. Br J Radiol 84:709-713, 2011

23. Putzier M, Hartwig T, Hoff EK, Streitparth F, Strube P: Minimally invasive TLIF leads to increased muscle sparing of the multifidus muscle but not the longissimus muscle compared with conventional PLIF-a prospective randomized clinical trial. Spine J 16:811-819, 2016

24. Rampersaud YR, Annand N, Dekutoski MB: Use of minimally invasive surgical techniques in the management of thoracolumbar trauma: current concepts. Spine (Phila Pa 1976) 31 (11 Suppl):S96-S104, 2006

25. Ringel F, Stoffel M, Stüer C, Meyer B: Minimally invasive transmuscular pedicle screw fixation of the thoracic and lum- bar spine. Neurosurgery 59 (4 Suppl 2):ONS361-ONS367, 2006

26. Scheer JK, Bakhsheshian J, Fakurnejad S, Oh T, Dahdaleh NS, Smith ZA: Evidence-based medicine of traumatic thoracolumbar burst fractures: a systematic review of operative management across 20 years. Global Spine J 5:73-82, 2015

27. Stavridis SI, Bücking P, Schaeren S, Jeanneret B, Schnake $\mathrm{KJ}$ : Implant removal after posterior stabilization of the thoraco-lumbar spine. Arch Orthop Trauma Surg 130:119-123, 2010

28. Takayama K, Kita T, Nakamura H, Kanematsu F, Yasunami $\mathrm{T}$, Sakanaka H, et al: New predictive index for lumbar paraspinal muscle degeneration associated with aging. Spine (Phila Pa 1976) 41:E84-E90, 2016

\section{Disclosures}

Dr. Steib is a consultant for and patent holder with Clariance. He has performed statistical analysis for a study and/or writing or editorial assistance on a manuscript for LDR, and he is also a patent holder with LDR.

\section{Author Contributions}

Conception and design: Charles. Acquisition of data: Ntilikina, Bahlau, Garnon, Charles. Analysis and interpretation of data: Ntilikina, Bahlau. Drafting the article: Ntilikina. Statistical analysis: Schaeffer. Study supervision: Schuller, Walter, Steib, Charles.

\section{Supplemental Information}

Previous Presentations

Deutscher Wirbelsäuberlenkongress (DWG), Frankfurt am Main, Germany, December 10-12, 2015; and Eurospine, Copenhagen, Denmark, September 4-6, 2015.

\section{Correspondence}

Yves Ntilikina, Service de Chirurgie du Rachis, Hôpitaux Universitaires de Strasbourg, Fédération de Médecine Translationnelle, Université de Strasbourg, 1 Place de l'Hôpital, B.P. 426, Strasbourg Cedex 67091, France. email: yves.ntilikina@ chru-strasbourg.fr. 